\title{
The clinical impact of preformed human leukocyte antigen-DQ donor-specific antibodies on graft outcomes in kidney transplantation
}

\author{
Sua Lee ${ }^{1}$, Byung Ha Chung ${ }^{2}$, Chul Woo Yang ${ }^{2}$ \\ ${ }^{1}$ Division of Nephrology, Department of Internal Medicine, Seoul National University Hospital, Seoul, Korea \\ ${ }^{2}$ Division of Nephrology, Department of Internal Medicine, Seoul St. Mary's Hospital, College of Medicine, The Catholic University of Korea, Seoul, Korea
}

Background: De-novo human leukocyte antigen (HLA)-DQ donor-specific antibody (DSA) is well known as a risk factor for graft dysfunction and graft loss in kidney transplantation (KT). Recently, the influence of preformed HLA-DQ DSA has been discussed. This study aimed to investigate the clinical impact of preformed HLA-DQ DSA on graft outcomes.

Methods: We evaluated 1,303 recipients who underwent KT at Seoul St. Mary's Hospital from January 2010 to December 2018. ABO incompatible KT was excluded. Finally, 990 recipients were included. According to the result of DSA using Luminex single antigen bead assay, recipients were classified as no DSA, only DQ, and only non-DQ groups. Primary outcome was biopsy-proven acute antibody-mediated rejection (AMR).

Results: Recipients were classified as no DSA (903 recipients, 91.2\%), only DQ (23 recipients, 2.3\%) and only non-DQ (56 recipients, $5.7 \%$ ). The incidence of acute rejection in only $D Q$ and only non-DQ groups were significantly higher than in no DSA group $(P=0.047$ and $P=0.021$, respectively). Especially, the incidence of acute $A M R$ in only $D Q$ and only non-DQ groups were significantly higher than in no DSA group ( $P=0.028$ and $P<0.001$, respectively). In logistic regression analysis, the presence of anti-HLA DQ DSA was presented as a risk factor for increased incidence of acute AMR (hazard ratio, 1.72; $P=0.029$ ). In Kaplan-Meier analysis, the cumulative incidences of acute AMR in only DQ and only non-DQ groups were significantly higher than in no DSA group $(P=0.010$ and $P<0.001$, respectively). There were no significant differences in graft loss and mortality.

Conclusions: Preformed anti-HLA DQ DSA could affect the development of acute rejection, especially acute AMR, as much as anti-HLA A, B and DR DSA. Therefore, the identification of preformed HLA-DQ DSA can help improve graft outcomes.

Corresponding author: Sua Lee

E-mail: soulmysoul27@naver.com

(c) The Korean Society for Transplantation

This is an Open Access article distributed under the terms of the Creative Commons Attribution Non-Commercial License (http://creativecommons.org/licenses/by-nc/4.0/) which permits unrestricted non-commercial use, distribution, and reproduction in any medium, provided the original work is properly cited. 